\title{
Nonlinear differential equations with perturbed Dirichlet integral boundary conditions
}

\author{
Alberto Cabada ${ }^{1 *}$ (D) and Javier Iglesias ${ }^{1}$
}

\author{
"Correspondence: \\ alberto.cabada@usc.gal \\ ${ }^{1}$ Departamento de Estatística, \\ Análise Matemática e Optimización \\ Instituto de Matemáticas, Facultade \\ de Matemáticas, Universidade de \\ Santiago de Compostela, Santiago \\ de Compostela, Galicia, Spain
}

\begin{abstract}
This paper is devoted to prove the existence of positive solutions of a second order differential equation with a nonhomogeneous Dirichlet conditions given by a parameter dependence integral. The studied problem is a nonlocal perturbation of the Dirichlet conditions by considering a homogeneous Dirichlet-type condition at one extreme of the interval and an integral operator on the other one. We obtain the expression of the Green's function related to the linear part of the equation and characterize its constant sign. Such a property will be fundamental to deduce the existence of solutions of the nonlinear problem. The results hold from fixed point theory applied to related operators defined on suitable cones.
\end{abstract}

MSC: 34B05; 34B08; 34B09; 34B10; 34B15; 34B18; 34B27

Keywords: Integral boundary conditions; Green's functions; Degree theory

\section{Introduction}

This paper is devoted to the study of the existence of solutions of the following family of nonlinear second order ordinary differential equations:

$$
u^{\prime \prime}(t)+\gamma u(t)+f(t, u(t))=0, \quad 0<t<1
$$

coupled to the following integral boundary conditions:

$$
u(0)=0, \quad u(1)=\lambda \int_{0}^{1} u(s) d s
$$

where $\gamma<\pi^{2}$ and $\lambda \in \mathbb{R}$.

To this end, we will distinguish the cases $\gamma=0, \gamma>0$ and $\gamma<0$. We will analyze each of them and give optimal sufficient conditions on $\gamma, \lambda$ and $f$ that allow us to ensure the existence of a solution of the considered problem.

This kind of problems model the behavior of an harmonic oscillator, subject to an external force $f$, which is fixed at the left extreme of the interval and has some mechanism at

C) The Author(s) 2021. This article is licensed under a Creative Commons Attribution 4.0 International License, which permits use, sharing, adaptation, distribution and reproduction in any medium or format, as long as you give appropriate credit to the original author(s) and the source, provide a link to the Creative Commons licence, and indicate if changes were made. The images or other third party material in this article are included in the article's Creative Commons licence, unless indicated otherwise in a credit line to the material. If material is not included in the article's Creative Commons licence and your intended use is not permitted by statutory regulation or exceeds the permitted use, you will need to obtain permission directly from the copyright holder. To view a copy of this licence, visit http://creativecommons.org/licenses/by/4.0/. 
the right one, that controls the displacement according to the feedback from devices measuring the displacements along parts of the oscillator. Integral boundary conditions have been considered in much work in the literature; see, for instance, $[6,9,12]$ (for second and fourth order ordinary differential equations) or $[1,5,7,8]$ (for fractional equations) and the references therein.

We refer a function $u \in \mathcal{C}([0,1])$ as a nonnegative solution of problem (1)-(2) if $u$ solves such problem and $u(t) \geq 0$, for all $t \in[0,1]$. A function $u \in \mathcal{C}([0,1])$ is called positive solution of problem (1)-(2) if it is a nonnegative solution and $u(t)>0$, for all $t \in(0,1)$.

The paper is organized as follows: in Sect. 2, we study the linear part of problem (1)(2), where we obtain the explicit expression of the related Green's function and calculate the exact values of $\gamma$ and $\lambda$ for which the Green's function has constant sign. In the next section we prove the existence of positive solutions for the nonlinear problem (1)-(2). Such solutions are given as the fixed points of a related integral operator defined on a suitable cone. At the end of this section we show two examples where the applicability of the obtained results is pointed out.

The following concept will be fundamental in order to deduce our existence results.

Definition 1 Let $X$ be a Banach space. A subset $K \subset X$ is a cone if:

- $K$ is closed,

- $K+K \subset K, \lambda K \subset K$ for all $\lambda \geq 0$ and $K \cap(-K)=\{0\}$.

We will use the celebrated expansion/contraction theorem of Krasnosels'kii [10].

Theorem 2 (Krasnosels'kii) Let $X$ be a Banach space and $K \subset X$ a cone in $X$. Let $\Omega_{1}, \Omega_{2} \subset$ $X$ open bounded such that $0 \in \Omega_{1} \subset \overline{\Omega_{1}} \subset \Omega_{2}$ and $T: K \cap\left(\overline{\Omega_{2}} \backslash \Omega_{1}\right) \rightarrow K$ a compact operator that satisfies one of the following properties:

1. $\|T(u)\| \geq\|u\|, \forall u \in K \cap \partial \Omega_{1}$ and $\|T(u)\| \leq\|u\|, \forall u \in K \cap \partial \Omega_{2}$.

2. $\|T(u)\| \leq\|u\|, \forall u \in K \cap \partial \Omega_{1}$ and $\|T(u)\| \geq\|u\|, \forall u \in K \cap \partial \Omega_{2}$.

Then $T$ has a fixed point at $K \cap\left(\overline{\Omega_{2}} \backslash \Omega_{1}\right)$.

\section{Linear part: Green's function}

In this section we obtain the expression of the related Green's function of the linear part of problem (1)-(2) and deduce some important properties that will be fundamental to obtain the existence of positive solutions of the nonlinear problem. To this end, we consider separately three cases depending on the sign of the real parameter $\gamma$. To do this, we will follow a treatment of a similar problem studied in [5] for fractional equations.

\subsection{Case $\gamma=0$}

In this subsection, we obtain the expression of the Green's function related to the linear problem

$$
u^{\prime \prime}(t)+\sigma(t)=0, \quad 0<t<1,
$$

coupled to the boundary conditions (2). 
Theorem 3 Let $\lambda \neq 2$ and $\sigma \in \mathcal{C}([0,1])$, then problem (3), (2) has a unique solution $u \in$ $\mathcal{C}^{2}([0,1])$, which is given by the following expression:

$$
u(t)=\int_{0}^{1} G(t, s) \sigma(s) d s
$$

where

$$
G(t, s)= \begin{cases}\frac{t(1-s)(2-\lambda+\lambda s)-(2-\lambda)(t-s)}{2-\lambda}, & 0 \leq s \leq t \leq 1, \\ \frac{t(1-s)(2-\lambda+\lambda s)}{2-\lambda}, & 0 \leq t<s \leq 1 .\end{cases}
$$

Proof By using the fundamental theorem of integral calculus to Eq. (3), together with Fubini's theorem, we arrive at the following expression:

$$
u(t)=-\int_{0}^{t}(t-s) \sigma(s) d s+c_{1} t+c_{2}
$$

Since $u(0)=0$, we deduce that $c_{2}=0$.

Now, the boundary condition at $t=1$ implies that

$$
\lambda \int_{0}^{1} u(s) d s=u(1)=-\int_{0}^{1}(1-s) \sigma(s) d s+c_{1}
$$

so

$$
c_{1}=\int_{0}^{1}(1-s) \sigma(s) d s+\lambda \int_{0}^{1} u(s) d s
$$

As a consequence

$$
u(t)=-\int_{0}^{t}(t-s) \sigma(s) d s+t \int_{0}^{1}(1-s) \sigma(s) d s+\lambda t \int_{0}^{1} u(s) d s
$$

By denoting $A=\int_{0}^{1} u(s) d s$, we have

$$
A=\int_{0}^{1} u(t) d t=-\int_{0}^{1} \int_{0}^{t}(t-s) \sigma(s) d s d t+\int_{0}^{1} \int_{0}^{1} t(1-s) \sigma(s) d s d t+\lambda A \int_{0}^{1} t d t
$$

Thus,

$$
\begin{aligned}
A & =-\int_{0}^{1} \int_{s}^{1}(t-s) \sigma(s) d t d s+\int_{0}^{1} \int_{0}^{1} t(1-s) \sigma(s) d t d s+\frac{\lambda}{2} A \\
& =-\int_{0}^{1} \frac{(1-s)^{2}}{2} \sigma(s) d s+\int_{0}^{1} \frac{(1-s)}{2} \sigma(s) d s+\frac{\lambda}{2} A .
\end{aligned}
$$

Then

$$
\begin{aligned}
A & =\frac{2}{2-\lambda}\left(-\int_{0}^{1} \frac{(1-s)^{2}}{2} \sigma(s) d s+\int_{0}^{1} \frac{(1-s)}{2} \sigma(s) d s\right) \\
& =-\frac{1}{2-\lambda} \int_{0}^{1}(1-s)^{2} \sigma(s) d s+\frac{1}{2-\lambda} \int_{0}^{1}(1-s) \sigma(s) d s .
\end{aligned}
$$


Substituting this value in (5), we obtain the expression of the solution $u$ as follows:

$$
\begin{aligned}
u(t)= & -\int_{0}^{t}(t-s) \sigma(s) d s+t \int_{0}^{1}(1-s) \sigma(s) d s-\frac{\lambda}{2-\lambda} t \int_{0}^{1}(1-s)^{2} \sigma(s) d s \\
& +\frac{\lambda}{2-\lambda} t \int_{0}^{1}(1-s) \sigma(s) d s \\
= & -\int_{0}^{t}(t-s) \sigma(s) d s+t \int_{0}^{1} \frac{(1-s)(2+\lambda(s-1))}{2-\lambda} \sigma(s) d s \\
= & \int_{0}^{t} \frac{t(1-s)(2+\lambda s-\lambda))-(2-\lambda)(t-s)}{2-\lambda} \sigma(s) d s \\
& +\int_{t}^{1} \frac{t(1-s)(2+\lambda s-\lambda))}{2-\lambda} \sigma(s) d s=\int_{0}^{1} G(t, s) \sigma(s) d s .
\end{aligned}
$$

In the sequel, we will state two lemmas related to the properties of the Green's function that will be useful to prove the existence of a positive solution of the nonlinear problem (1)-(2) with $\gamma=0$.

Lemma 4 Let $G$ be the Green's function related to problem (3), (2), given by Eq. (4). Then, for all $\lambda \neq 2$, the following properties are fulfilled:

1. $G(0, s)=G(t, 0)=G(t, 1)=0$, for all $t, s \in[0,1]$.

2. $G(t, s)$ is continuous on $[0,1] \times[0,1]$.

3. $G(1, s)=0$ for all $s \in(0,1)$ if and only if $\lambda=0$.

4. $(2-\lambda) G(1, s)>0$ for all $s \in(0,1)$ if and only if $\lambda>0$.

5. $(2-\lambda) G(s, s)>0$ for all $s \in(0,1)$ if and only if $\lambda<2$.

6. $G(t, s)>0$ for all $t, s \in(0,1)$ if and only if $\lambda \in[0,2)$.

7. $G(t, s)$ changes sign on $(0,1) \times(0,1)$ for all $\lambda \notin[0,2)$.

8. For all $\lambda \in[0,2), G(t, s) \leq \frac{1}{2(2-\lambda)}, \forall t, s \in[0,1]$.

Proof Properties 1. and 2. are immediate. Let us now prove the remaining properties:

3. Let $s \in(0,1)$, then $G(1, s)=0$ if and only if $\lambda s(1-s)=0$, i.e. $\lambda=0$.

4. The result holds trivially from the fact that

$$
(2-\lambda) G(1, s)=\lambda s(1-s), \quad \text { for all } s \in(0,1) .
$$

5. The result is immediately deduced from the following equality:

$$
(2-\lambda) G(s, s)=s(1-s)(2-\lambda(1-s)), \quad \text { for all } s \in(0,1) .
$$

6. Since $G(t, s)$ is linear on $t$, for all $s \in[0,1]$ fixed, $G(t, s)$ attains its maximum and minimum at $t=0, t=s$ or at $t=1$.

From Property 1., we have $G(0, s)=0$ for all $s \in[0,1]$.

From Property 5., we have $G(s, s)>0$ for all $s \in(0,1)$, if and only if $\lambda<2$.

From Property 4., we have $G(1, s)>0$ for all $s \in(0,1)$, if and only if $\lambda \in(0,2)$.

As a consequence of the three previous assertions and Property 3., this property holds. 
7. From (6) it is clear that $G(1, s)<0$ for all $s \in(0,1)$, if and only if $\lambda \notin(0,2)$.

From (7) it is clear that $G(s, s)>0$ for all $s \in(0,1)$ and $\lambda \leq 0$ and, moreover, if $\lambda>2$, we have $G(s, s)>0$ for $s \in(0,1)$ close enough to 0 . Thus, this property is fulfilled.

8. From Property 6., we know that $G(t, s)>0$ for all $(t, s) \in(0,1) \times(0,1)$. As in Property 6., we know that the maximum values will be attained at $G(s, s)$ and/or $G(1, s)$. Now, since

$$
(2-\lambda) G(s, s)=s(1-s)(2-\lambda(1-s))=2 s(1-s)-\lambda s(1-s)^{2} \leq 2 s(1-s) \leq \frac{1}{2}
$$

and

$$
(2-\lambda) G(1, s)=\lambda s(1-s) \leq \frac{\lambda}{4}<\frac{1}{2},
$$

the proof is concluded.

In the sequel, we deduce two sharp inequalities for the positiveness of the Green's function.

Lemma 5 Let $\lambda \in(0,2)$ and $G(t, s)$ be the Green's function related to problem (3), (2), given by Eq. (4). Then the following properties hold:

$$
t G(1, s) \leq G(t, s) \leq \frac{2}{\lambda} G(1, s), \quad \text { for all } s, t \in[0,1]
$$

Proof For $t=0 t=1, s=0$ or $s=1$ the inequalities follow immediately from Properties 1 . and 4 . in Lemma 4.

Now let $t, s$ be such that $0<t \leq s<1$. In this case

$$
h(t, s):=\frac{G(t, s)}{G(1, s)}=\frac{t(2-\lambda(1-s))}{\lambda s}=t\left(1+\frac{2-\lambda}{\lambda s}\right) .
$$

As a consequence, we have

$$
t<t\left(1+\frac{2-\lambda}{\lambda s}\right)=h(t, s) \leq t \frac{2}{\lambda s}=\frac{2}{\lambda} \frac{t}{s} \leq \frac{2}{\lambda} .
$$

Consider now the case $0<s \leq t<1$, using $s \geq t$, we conclude that

$$
\begin{aligned}
h(t, s) & =\frac{t(1-s)(2-\lambda(1-s))-(2-\lambda)(t-s)}{\lambda s(1-s)} \\
& \geq \frac{t(1-s)(2-\lambda(1-s))-(2-\lambda)(t-t s)}{\lambda s(1-s)} \\
& =\frac{t(1-s)[2-\lambda(1-s)-(2-\lambda)]}{\lambda s(1-s)}=t .
\end{aligned}
$$

Moreover,

$$
\lim _{t \rightarrow s^{+}} h(t, s)=\frac{2-\lambda(1-s)}{\lambda} \leq \frac{2}{\lambda}
$$


and

$$
\lim _{t \rightarrow 1^{-}} h(t, s)=\frac{(1-s)[(2-\lambda(1-s))-(2-\lambda)]}{\lambda s(1-s)}=1 .
$$

On the other hand, since $h(t, s)$ is a nonnegative, continuous and linear function with respect to $t$, we deduce

$$
t \leq h(t, s) \leq \max \left\{\lim _{t \rightarrow 1^{-}} h(t, s), \lim _{t \rightarrow s^{+}} h(t, s)\right\}=\frac{2}{\lambda} .
$$

From Property 4. in Lemma 4, we conclude that the inequalities (8) are satisfied.

\subsection{Case $\gamma>0$}

In this subsection, we will obtain the expression of the Green's function related to the problem

$$
u^{\prime \prime}(t)+m^{2} u(t)+\sigma(t)=0, \quad 0<t<1
$$

coupled to the boundary conditions (2).

Theorem 6 Let $\lambda \neq \frac{m \sin m}{1-\cos m}, m>0, m \neq 2 k \pi, k=1,2, \ldots$, and $\sigma \in \mathcal{C}([0,1])$. Then problem (9), (2) has a unique solution $u \in \mathcal{C}^{2}([0,1])$, which is given by

$$
u(t)=\int_{0}^{1} G_{m}(t, s) \sigma(s) d s
$$

where

$$
G_{m}(t, s)= \begin{cases}G_{m}^{1}(t, s), & 0 \leq s \leq t \leq 1 \\ G_{m}^{2}(t, s), & 0 \leq t<s \leq 1\end{cases}
$$

Here, if $m \neq k \pi, k \in \mathbb{N}$ odd,

$$
\begin{aligned}
G_{m}^{1}(t, s)= & \frac{\sin (m s)[\sin (m-m t)(m \sin m-\lambda(1-\cos m))+\lambda \sin (m t)]}{m \sin m(m \sin m-\lambda(1-\cos m))} \\
& +\frac{\lambda \sin (m t)(\sin (m-m s)-\sin m)}{m \sin m(m \sin m-\lambda(1-\cos m))}
\end{aligned}
$$

and

$$
G_{m}^{2}(t, s)=\frac{\sin (m t)[\sin (m-m s)(m \sin m+\lambda \cos m)+\lambda(\sin (m s)-\sin m)]}{m \sin m(m \sin m-\lambda(1-\cos m))},
$$

and, if $m=k \pi$, for some $k \in \mathbb{N}$ odd,

$$
G_{k \pi}^{1}(t, s)=\frac{2 \lambda \sin (\pi k s) \cos (\pi k t)+\sin (\pi k t)(\lambda(-\cos (\pi k s))-\pi k \sin (\pi k s)+\lambda)}{2 \pi k \lambda}
$$

and

$$
G_{k \pi}^{2}(t, s)=\frac{\sin (\pi k t)(\lambda \cos (\pi k s)-\pi k \sin (\pi k s)+\lambda)}{2 \pi k \lambda} .
$$


Proof It is immediate to verify that the spectrum of problem (9), (2) is given by the following pairs on the plane $(m, \lambda)$ :

1. $\left(m, \frac{m \sin m}{1-\cos m}\right), m>0, m \neq 2 k \pi, k=1,2, \ldots$

2. $(2 k \pi, \lambda), \lambda \in \mathbb{R}, k=1,2, \ldots$

Consider the case $m \neq k \pi, k \in \mathbb{N}$ odd, and let $v$ be the unique solution of

$$
\left\{\begin{array}{l}
v^{\prime \prime}(t)+m^{2} v(t)+\sigma(t)=0, \quad 0<t<1, \\
v(0)=0, \quad v(1)=0,
\end{array}\right.
$$

and $w$ be defined as the unique solution of

$$
\left\{\begin{array}{l}
w^{\prime \prime}(t)+m^{2} w(t)=0, \quad 0<t<1, \\
w(0)=0, \quad w(1)=1,
\end{array}\right.
$$

then it is not difficult to verify that $u(t)=v(t)+\left(\lambda \int_{0}^{1} u(s) d s\right) w(t)$ is a solution of (9), (2).

In [3] there has been constructed and algorithm to calculate the exact expression of the Green's function related to any $n$th order differential equation, with constant coefficients coupled to arbitrary two-point linear boundary conditions. Such algorithm has been developed in a Mathematica package that is available in [4]. So, using this package, we obtain

$$
v(t)=\int_{0}^{1} G_{m}^{v}(t, s) \sigma(s) d s
$$

where

$$
G_{m}^{v}(t, s)= \begin{cases}\frac{\sin (m s) \sin (m-m t)}{m \sin m}, & 0 \leq s \leq t \leq 1, \\ \frac{\sin (m-m s) \sin (m t)}{m \sin m}, & 0 \leq t<s \leq 1 .\end{cases}
$$

It is immediate to verify that

$$
w(t)=\frac{\sin (m t)}{\sin m} .
$$

Thus,

$$
u(t)=\int_{0}^{1} G_{m}^{v}(t, s) \sigma(s) d s+\frac{\lambda}{\sin m}\left(\int_{0}^{1} u(s) d s\right) \sin (m t) .
$$

Denoting $A=\int_{0}^{1} u(s) d s$, we deduce from the previous expression that

$$
\begin{aligned}
A & =\int_{0}^{1} u(t) d t \\
& =\int_{0}^{1} \int_{0}^{1} G_{m}^{v}(t, s) \sigma(s) d s d t+\frac{\lambda}{\sin m} A \int_{0}^{1} \sin (m t) d t \\
& =\int_{0}^{1} \int_{0}^{t} \frac{\sin (m s) \sin (m-m t)}{m \sin m} \sigma(s) d s d t+\int_{0}^{1} \int_{t}^{1} \frac{\sin (m-m s) \sin (m t)}{m \sin m} \sigma(s) d s d t
\end{aligned}
$$




$$
\begin{aligned}
& +\frac{\lambda}{\sin m} A \int_{0}^{1} \sin (m t) d t=\int_{0}^{1} \frac{\sin (m s)(1-\cos (m-m s))}{m^{2} \sin m} \sigma(s) d s \\
& +\int_{0}^{1} \frac{\sin (m-m s)(1-\cos (m s))}{m^{2} \sin m} \sigma(s) d s+A \frac{\lambda}{\sin m} \frac{1-\cos m}{m} .
\end{aligned}
$$

As a consequence, it follows that

$$
\begin{aligned}
A & =\left(1-\frac{\lambda(1-\cos m)}{m \sin m}\right)^{-1} \int_{0}^{1} \frac{\sin (m s)+\sin (m-m s)-\sin m}{m^{2} \sin m} \sigma(s) d s \\
& =\int_{0}^{1} \frac{\sin (m s)+\sin (m-m s)-\sin m}{m(m \sin m-\lambda(1-\cos m))} \sigma(s) d s .
\end{aligned}
$$

Substituting this value in (11) we obtain the following expression:

$$
\begin{aligned}
u(t)= & \int_{0}^{t} \frac{\sin (m s) \sin (m-m t)}{m \sin m} \sigma(s) d s+\int_{t}^{1} \frac{\sin (m-m s) \sin (m t)}{m \sin m} \sigma(s) d s \\
& +\int_{0}^{1} \frac{\sin (m s)+\sin (m-m s)-\sin m}{m(m \sin m-\lambda(1-\cos m))} \frac{\lambda \sin (m t)}{\sin m} \sigma(s) d s \\
= & \int_{0}^{t}\left(\frac{\sin (m s)[\sin (m-m t)(m \sin m-\lambda(1-\cos m))+\lambda \sin (m t)]}{m \sin m(m \sin m-\lambda(1-\cos m))}\right. \\
& \left.+\frac{\lambda \sin (m t)(\sin (m-m s)-\sin m)}{m \sin m(m \sin m-\lambda(1-\cos m))}\right) \sigma(s) d s \\
& +\int_{t}^{1} \frac{\sin (m t)[\sin (m-m s)(m \sin m+\lambda \cos m)+\lambda(\sin (m s)-\sin m)]}{m \sin m(m \sin m-\lambda(1-\cos m))} \sigma(s) d s \\
= & \int_{0}^{1} G_{m}(t, s) \sigma(s) d s .
\end{aligned}
$$

The uniqueness of the Green's function is deduced from the uniqueness of functions $v$ and $w$.

The expressions of the Green's function for $m=k \pi$, with $k \in \mathbb{N}$ odd, follow by taking the limit of the expressions of $G_{m}^{1}$ and $G_{m}^{2}$ when $m$ goes to $k \pi$. By direct calculations, it is immediate to verify that such function satisfies the properties of the Green's function of problem (9), (2) with $m=k \pi, k \in \mathbb{N}$ odd.

In the same way as in the case $\gamma=0$, we will now state different results about the properties of the Green's function that we have just obtained. As before, we will try to give conditions that allow us to ensure that the Green's function attains a constant sign. To this end, we will use the following property, which is a direct consequence of the Sturm comparison theorem

Remark 7 Since $w(t)=\sin (\pi t)$, is a nontrivial solution of

$$
w^{\prime \prime}(t)+\pi^{2} w(t)=0
$$

and it vanishes in $\mathbb{Z}$, from the classical Sturm comparison result, we see that for $\gamma<\pi^{2}$ any nontrivial solution of the problem

$$
v^{\prime \prime}(t)+\gamma v(t)=0
$$


vanishes at most once in the interval $[0,1]$. Therefore, the distance between two zeros of any nontrivial solution must be greater than 1 .

Lemma 8 Let $G_{m}$ be the Green's function related to problem (9), (2), given by Eq. (10). Then, for all $\lambda \neq \frac{m \sin m}{1-\cos m}, m>0, m \neq 2 k \pi, k=1,2, \ldots$, the following properties hold:

1. $G_{m}(0, s)=G_{m}(t, 1)=G_{m}(t, 0)=0$, for all $t, s \in[0,1]$.

2. $G_{m}(t, s)$ is continuous at $(t, s) \in[0,1] \times[0,1]$.

3. If $m \in(0,2 \pi)$, then $G_{m}(1, s)=0$ for all $s \in(0,1)$ if and only if $\lambda=0$.

4. If $m \in(0,2 \pi)$, then $(m \sin m-\lambda(1-\cos m)) G_{m}(1, s)>0$ for all $s \in(0,1)$ and $\lambda>0$.

5. $G_{m}(t, s)>0$ for all $t, s \in(0,1)$ if and only if $0 \leq \lambda<\frac{m \sin m}{1-\cos m}, m \in(0, \pi]$.

6. $G_{m}(t, s)$ changes sign on $(0,1) \times(0,1)$ for all $\lambda \notin\left[0, \frac{m \sin m}{1-\cos m}\right), m \in(0, \pi]$.

Proof Properties 1. and 2. are immediate. Let us now consider the others:

3. Let $s \in(0,1)$, then $G_{m}(1, s)=0$ if and only if

$$
\frac{\lambda(\sin (m s)+\sin (m-m s)-\sin m)}{m(m \sin m-\lambda(1-\cos m))}=0
$$

which is equivalent to

$$
\lambda(\sin (m s)+\sin (m-m s)-\sin m)=: \lambda r_{m}(s)=0 .
$$

It is easy to check that if $m \in(0,2 \pi)$ then function $r_{m}$ has a unique maximum at $s=\frac{1}{2}$ and, since $r_{m}(0)=0=r_{m}(1)$, we deduce that $r_{m}(s)>0$ for $s \in(0,1)$. Therefore $G_{m}(1, s)=0$ if and only if $\lambda=0$.

4. From the previous assertion, we have

$$
(m \sin m-\lambda(1-\cos m)) G_{m}(1, s)=\frac{\lambda}{m} r_{m}(s)>0,
$$

for all $s \in(0,1), m \in(0,2 \pi)$ and $\lambda>0$.

5. Assume now that $0 \leq \lambda<\frac{m \sin m}{1-\cos m}, m \in(0, \pi]$. Using Properties 1 . and 4., we know that, for each $s \in(0,1), G_{m}(0, s)=0$ and $G_{m}(1, s) \geq 0$. In addition,

$$
\frac{\partial G_{m}}{\partial t}(0, s)=\frac{\sin (m(1-s))}{\sin m}+\frac{\lambda r_{m}(s)}{\sin m(m \sin m+\lambda(1-\cos m))}>0 .
$$

Therefore, $G_{m}(t, s)$ is increasing and positive to the right of $t=0$ for every $s \in(0,1)$.

On the other hand,

$$
\frac{\partial^{2} G_{m}}{\partial t^{2}}(t, s)+m^{2} G_{m}(t, s)=0, \quad t \in[0,1], t \neq s .
$$

Since $G_{m}(\cdot, s) \in \mathcal{C}^{2}([0, s) \cup(s, 1])$, we can use Remark 7 on each interval of the form $\left[0, s_{0}\right] \subset[0, s)$ and $\left[s_{1}, 1\right] \subset(s, 1]$ (notice that we cannot apply such a remark to the whole interval $[0,1]$, because $\left.G_{m}(\cdot, s) \notin \mathcal{C}^{2}([0,1])\right)$.

Suppose that there exists $t_{0} \in(0,1)$ such that $G_{m}\left(t_{0}, s\right)=0$. We distinguish the following cases:

- If $t_{0} \in[0, s)$, since $G_{m}(0, s)=0$ and $G_{m}(\cdot, s) \in \mathcal{C}^{2}\left(\left[0, t_{0}\right]\right)$, we would have two zeros that distances less than 1 , which is not possible. 
- Now suppose $t_{0}=s$. In this case, since $\left.\frac{\partial^{2}}{\partial t^{2}} G_{m}(t, s)\right|_{t=s^{-}}$exists and is finite, we can extend $G_{m}(\cdot, s)$ as a $\mathcal{C}^{2}$ function on the interval $[0, s]$, which brings us back to the previous case.

- If $t_{0} \in(s, 1)$, considering the $\mathcal{C}^{2}$ extension to interval $[s, 1]$, since $G_{m}(s, s)>0$ and $G_{m}(1, s) \geq 0$, can occur three situations:

(a) If $\frac{\partial G_{m}}{\partial t}\left(t_{0}, s\right)<0$, then there exists $t_{1} \in\left(t_{0}, 1\right]$ such that $G_{m}\left(t_{1}, s\right)=0$, which is not possible as a consequence of Remark 7 applied on $[s, 1]$.

(b) If $\frac{\partial G_{m}}{\partial t}\left(t_{0}, s\right)=0$, since $G_{m}(\cdot, s)$ is a solution of (12) on $[s, 1]$, we deduce that $G_{m}(t, s) \equiv 0$ in $[s, 1]$, which contradicts the fact that $G_{m}(s, s)>0$.

(c) If $\frac{\partial G_{m}}{\partial t}\left(t_{0}, s\right)>0$, then there exists $t_{1} \in\left(s, t_{0}\right)$ such that $G\left(t_{1}, s\right)=0$, reaching a contradiction again.

From the equality

$$
(m \sin m-\lambda(1-\cos m)) G_{m}(1, s)=\frac{\lambda}{m} r_{m}(s),
$$

we deduce immediately that $G_{m}(1, s)$ takes positive and negative values for all $m>2 \pi, m \neq 2 k \pi, k=1,2, \ldots$, and all $\lambda \in \mathbb{R}, \lambda \neq 0$. Moreover, from the expression of $G_{m}(1, s)$ we deduce that if either, $\lambda<0$ and $m \in(0,2 \pi)$, or $\lambda>\frac{m \sin m}{1-\cos m}$ and $m \in(0, \pi]$, or $\lambda>0$ and $m \in(\pi, 2 \pi)$, then $G_{m}(1, s)<0$.

6. Using the previous assertion, we only need to verify that if $\lambda \notin\left[0, \frac{m \sin m}{1-\cos m}\right)$, $m \in(0, \pi]$, then $G_{m}(t, s)$ takes some positive values on $(0,1) \times(0,1)$. But to verify this property it is enough to consider function

$$
f_{m}(t):=G_{m}(t, t)
$$

By direct computation, we have $f_{m}^{\prime}(0)=1$ for all $\lambda \in \mathbb{R}$ and $m>0, m \neq 2 k \pi$, $k=1,2, \ldots$. In consequence, $G_{m}(t, t)>0$ in a small enough neighborhood of $(0,0)$.

Now we deduce the following stronger condition on the Green's function.

Lemma 9 Let $0<m<\pi, 0 \leq \lambda<\frac{m \sin m}{1-\cos m}$ and $G_{m}(t, s)$ be the Green's function of problem (9), (2) given by Eq. (10). Then there are $h_{1} \in \mathcal{C}([0,1]), h_{1}>0$ on $(0,1]$ and $C_{1} \in \mathbb{R}, C_{1}>0$ such that

$$
h_{1}(t) G_{m}(t, s) \leq G_{m}(t, s) \leq C_{1} G_{m}(1, s), \quad \text { for all } t, s \in[0,1] .
$$

Proof If $t=0, s=0$ or $s=1$ the result follows from Lemma 8. Let then $0<t \leq 1$ be arbitrarily set. Since

$$
\begin{aligned}
& \lim _{s \rightarrow 0^{+}} \frac{G_{m}(t, s)}{G_{m}(1, s)}=\frac{\sin (m-m t)(m \sin m-\lambda(1-\cos m))}{\lambda \sin m(1-\cos m)}>0, \\
& \lim _{s \rightarrow 1^{-}} \frac{G_{m}(t, s)}{G_{m}(1, s)}=1,
\end{aligned}
$$

and taking into account the Properties 4 . and 5 . of Lemma 8 , then, for any $s \in(0,1)$ fixed, we can extend function $\frac{G_{m}(\cdot, s)}{G_{m}(1, s)}$ continuously to the interval $[0,1]$ and, furthermore, this extension is strictly positive for all $t \in(0,1]$. 
As a consequence,

$$
h_{1}(t)=\min _{s \in[0,1]} \frac{G_{m}(t, s)}{G_{m}(1, s)}>0, \quad \text { for all } t \in(0,1],
$$

and

$$
C_{1}=\max _{t \in[0,1]}\left\{\max _{s \in[0,1]} \frac{G_{m}(t, s)}{G_{m}(1, s)}\right\} \geq 1
$$

from which the result follows.

\subsection{Case $\boldsymbol{\gamma}<0$}

Next, we will give the expression of the Green's function of the problem

$$
u^{\prime \prime}(t)-m^{2} u(t)+\sigma(t)=0, \quad 0<t<1
$$

coupled to the boundary conditions (2).

In this section we will omit most of the proofs because they are analogous to those presented in previous cases.

First, we will state a lemma that will be useful for the calculation of this function.

Lemma 10 ([2, Appendix B]) Let us consider the problem

$$
(P) \quad\left\{\begin{array}{l}
u^{\prime \prime}(t)-m^{2} u(t)+\sigma(t)=0 \\
u(0)=0=u(1)
\end{array}\right.
$$

The Green's function associated with problem $(P)$ is given by the following expression:

$$
G_{m}(t, s)= \begin{cases}\frac{\sinh (m s) \sinh (m(1-t))}{m \sinh m}, & 0 \leq s \leq t \leq 1, \\ \frac{\sinh (m t) \sinh (m(1-s))}{m \sinh m}, & 0 \leq t<s \leq 1\end{cases}
$$

Theorem 11 Let $\lambda \neq \frac{m \sinh m}{\cosh m-1}$ and $\sigma \in \mathcal{C}([0,1])$, then problem (14), (2) has a unique solution $u \in \mathcal{C}^{2}([0,1])$, which is given by the expression

$$
u(t)=\int_{0}^{1} G_{m}(t, s) \sigma(s) d s
$$

where

$$
G_{m}(t, s)= \begin{cases}G_{m}^{1}(t, s), & 0 \leq s \leq t \leq 1 \\ G_{m}^{2}(t, s), & 0 \leq t<s \leq 1\end{cases}
$$

with

$$
\begin{aligned}
G_{m}^{1}(t, s)= & \frac{\sinh (m s)[\sinh (m-m t)(m \sinh m+\lambda(1-\cosh m))-\lambda \sinh (m t)]}{m \sinh m(m \sinh m+\lambda(1-\cosh m))} \\
& -\frac{\lambda \sinh (m t)(\sinh (m-m s)-\sinh m)}{m \sinh m(m \sinh m+\lambda(1-\cosh m))}
\end{aligned}
$$


and

$$
G_{m}^{2}(t, s)=\frac{\sinh (m t)[\sinh (m-m s)(m \sinh m-\lambda \cosh m)-\lambda(\sinh (m s)-\sinh m)]}{m \sinh m(m \sinh m+\lambda(1-\cosh m))} .
$$

Proof In a similar way to Theorem 6 and using Lemma 10, we construct the Green's function taking into account that we can express the solution as

$$
u(t)=\int_{0}^{1} G_{m}(t, s) \sigma(s) d s+\lambda\left(\int_{0}^{1} u(s) d s\right) w(t)
$$

where $w(t)=\frac{\sinh m t}{\sinh m}$ is the unique solution of the following problem:

$$
\left\{\begin{array}{l}
w^{\prime \prime}(t)-m^{2} w(t)=0, \quad 0<t<1 \\
w(0)=0, \quad w(1)=1 .
\end{array}\right.
$$

We will now enunciate some properties of $G_{m}$. The proofs are analogous to those that have been presented in the two previous cases.

Lemma 12 Let $G_{m}$ be the Green's function associated with problem (14), (2), given by Eq, (16). Then, for all $\lambda \neq \frac{m \sinh m}{\cosh m-1}, m>0$, the following properties hold:

1. $G_{m}(0, s)=G_{m}(t, 1)=G_{m}(t, 0)=0$, for all $t, s \in[0,1]$.

2. $G_{m}(t, s)$ is continuous on $[0,1] \times[0,1]$.

3. $G_{m}(1, s)=0$, for all $s \in(0,1)$ if and only if $\lambda=0$.

4. $(m \sinh m+\lambda(1-\cosh m)) G_{m}(1, s)>0$ for all $s \in(0,1)$ and $m>0$.

5. $G_{m}(t, s)>0$ for all $t, s \in(0,1)$ if and only if $0 \leq \lambda<\frac{m \sinh m}{\cosh m-1}, m>0$.

6. $G_{m}(t, s)$ changes sign on $(0,1) \times(0,1)$ for all $\lambda \notin\left[0, \frac{m \sinh m}{\cosh m-1}\right), m>0$.

Lemma 13 Let $m>0,0 \leq \lambda<\frac{m \sinh m}{\cosh m-1}$ and $G_{m}(t, s)$ be the Green's function of problem (14), (2) given by Eq. (16). Then there exist $h_{2} \in \mathcal{C}([0,1]), h_{2}>0$ in $(0,1]$ and $C_{2}>0$ such that

$$
h_{2}(t) G_{m}(1, s) \leq G_{m}(t, s) \leq C_{2} G_{m}(1, s), \quad \text { for all } t, s \in[0,1]
$$

\section{Nonlinear problem}

This section is devoted to proving the existence of positive solutions on $(0,1)$ of problem (1)-(2). We will assume the following regularity condition for the nonlinear part of the equation:

(f) $f:[0,1] \times[0, \infty) \rightarrow[0, \infty)$ is a continuous function.

As in the previous section, we will distinguish three different cases depending on the sign of the parameter $\gamma$. The results hold from the application of the Krasnosel'skil fixed point Theorem 2 to the operator $T_{\gamma}: \mathcal{C}([0,1]) \rightarrow \mathcal{C}([0,1])$ defined as

$$
T_{\gamma} u(t):=\int_{0}^{1} G_{\gamma}(t, s) f(s, u(s)) d s, \quad t \in[0,1]
$$

Here, $G_{\gamma}$ corresponds to the function $G$, given by (4), if $\gamma=0, G_{m}$ given by (10) if $\gamma=$ $m^{2}>0$, and $G_{m}$ given by (16) if $\gamma=-m^{2}<0$. 
As we have proved in previous section, we know that the fixed points of operator $T_{\gamma}$ coincide with the solutions of problem (1)-(2).

To apply Theorem 2 , we define $X=(\mathcal{C}([0,1]),\|\cdot\|)$, the Banach space endowed with the supremum norm.

Now, we denote

$$
f_{0}=\lim _{u \rightarrow 0^{+}}\left\{\min _{t \in\left[\frac{1}{2}, 1\right]} \frac{f(t, u)}{u}\right\}, \quad f_{\infty}=\lim _{u \rightarrow+\infty}\left\{\min _{t \in\left[\frac{1}{2}, 1\right]} \frac{f(t, u)}{u}\right\}
$$

and

$$
f^{0}=\lim _{u \rightarrow 0^{+}}\left\{\max _{t \in[0,1]} \frac{f(t, u)}{u}\right\} \text { and } f^{\infty}=\lim _{u \rightarrow+\infty}\left\{\max _{t \in[0,1]} \frac{f(t, u)}{u}\right\} .
$$

In the sequel, we introduce the cone $K_{\gamma} \subset X$, depending on the sign of the real parameter $\gamma$.

If $\gamma=0$ :

$$
K_{\gamma}=\left\{u \in X ; u(t) \geq 0, t \in[0,1], u(t) \geq t \frac{\lambda}{2}\|u\|, t \in[0,1]\right\} .
$$

If $\gamma>0$ :

$$
K_{\gamma}=\left\{u \in X ; u(t) \geq 0, u(t) \geq \frac{h_{1}(t)}{C_{1}}\|u\|, t \in[0,1]\right\} .
$$

If $\gamma<0$ :

$$
K_{\gamma}=\left\{u \in X ; u(t) \geq 0, u(t) \geq \frac{h_{2}(t)}{C_{2}}\|u\|, t \in[0,1]\right\} .
$$

Here $h_{1}$ and $C_{1}$ are given in Lemma 9, and $h_{2}$ and $C_{2}$ are given in Lemma 13.

So, we arrive at the following existence result.

Theorem 14 Let us consider problem (1)-(2), and let $\Delta:\left(-\infty, \pi^{2}\right) \rightarrow \mathbb{R}$ be the function defined as (see Fig. 1):

$$
\Delta(\gamma)= \begin{cases}\frac{\sqrt{-\gamma} \sinh (\sqrt{-\gamma})}{\cosh (\sqrt{-\gamma})-1}, & \gamma<0, \\ 2, & \gamma=0, \\ \frac{\sqrt{\gamma} \sin (\sqrt{\gamma})}{1-\cos (\sqrt{\gamma})}, & \gamma>0 .\end{cases}
$$

Suppose further that $(f)$ holds and one of the two following conditions is fulfilled:

(i) (sublinear case) $f_{0}=\infty$ and $f^{\infty}=0$.

(ii) (superlinear case) $f^{0}=0$ and $f_{\infty}=\infty$.

So, for all $\gamma<\pi^{2}$ and $0<\lambda<\Delta(\gamma)$ there is a positive solution of problem (1)-(2), $u \in K_{\gamma}$.

Proof Consider, to start with, the case $\gamma=m^{2}>0$.

Let us first see that $T: K_{\gamma} \rightarrow K_{\gamma}$ is a compact operator. 


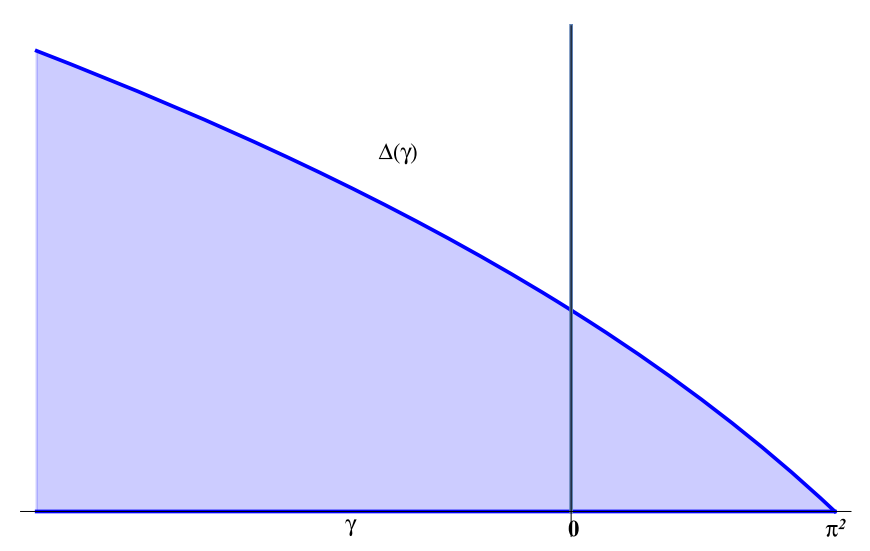

Figure 1 Graphic of $\Delta(\gamma)$

Since $G_{m}$ and $f$ are continuous and nonnegative in their domain of definition, we have $T_{\gamma} u \in \mathcal{C}([0,1])$ and $T_{\gamma} u(t) \geq 0$, for all $t \in[0,1]$.

Let $u \in K_{\gamma}$, using the properties stated in Lemmas 8 and 9, we have, for all $t \in[0,1]$,

$$
\begin{aligned}
T_{\gamma} u(t) & =\int_{0}^{1} G_{m}(t, s) f(s, u(s)) d s \geq \int_{0}^{1} h_{1}(t) G(1, s) f(s, u(s)) d s \\
& \geq \frac{h_{1}(t)}{C_{1}} \int_{0}^{1}\left\{\max _{t \in[0,1]} G(t, s)\right\} f(s, u(s)) d s \\
& \geq \frac{h_{1}(t)}{C_{1}} \max _{t \in[0,1]}\left\{\int_{0}^{1} G_{m}(t, s) f(s, u(s)) d s\right\} \\
& =\frac{h_{1}(t)}{C_{1}}\left\|T_{\gamma} u\right\| .
\end{aligned}
$$

Furthermore, the continuity of the functions $G_{m}$ and $f$ guarantees the continuity of the operator $T: K_{\gamma} \rightarrow K_{\gamma}$. So $T\left(K_{\gamma}\right) \subset K_{\gamma}$.

Let us now verify that the image by $T_{\gamma}$ of a bounded set is relatively compact. To this end, we will use the Arzelà-Ascoli theorem.

Let $\Omega \subset K_{\gamma}$ bounded, that is, there exists $M \in \mathbb{R}, M>0$ such that $\|u\| \leq M$, for all $u \in \Omega$. Let us define

$$
L=\max _{0 \leq t \leq 1,0 \leq u \leq M}|f(t, u)| .
$$

Then, for all $u \in \Omega$ and $t \in[0,1]$, we have

$$
\begin{aligned}
\left|T_{\gamma} u(t)\right| & =\left|\int_{0}^{1} G_{m}(t, s) f(s, u(s)) d s\right| \\
& \leq L \int_{0}^{1} G_{m}(t, s) d s \leq L \int_{0}^{1} C_{1} G_{m}(1, s) d s \\
& =L C_{1} \frac{\lambda(2-2 \cos m-m \sin m)}{m^{2}(m \sin m-\lambda(1-\cos m))}:=N .
\end{aligned}
$$


As a consequence,

$$
\left\|T_{\gamma} u\right\| \leq N
$$

so $T_{\gamma}(\Omega)$ is bounded.

On the other hand, for each $u \in \Omega$ and $t \in[0,1]$ we have

$$
\begin{aligned}
\left|\left(T_{\gamma} u\right)^{\prime}(t)\right| & =\left|\int_{0}^{1} \frac{\partial G_{m}}{\partial t}(t, s) f(s, u(s)) d s\right| \leq \int_{0}^{1}\left|\frac{\partial G_{m}}{\partial t}(t, s)\right||f(s, u(s))| d s \\
& \leq L \int_{0}^{1}\left|\frac{\partial G_{m}}{\partial t}(t, s)\right| d s=: N^{\prime}
\end{aligned}
$$

The regularity of the Green's function allows us to ensure that $N^{\prime} \in \mathbb{R}, N^{\prime}>0$. Thus, for all $t_{1}, t_{2} \in[0,1], t_{1}<t_{2}$, the following inequality is fulfilled:

$$
\left|\left(T_{\gamma} u\right)\left(t_{2}\right)-\left(T_{\gamma} u\right)\left(t_{1}\right)\right|=\left|\int_{t_{1}}^{t_{2}}\left(T_{\gamma} u\right)^{\prime}(s) d s\right| \leq \int_{t_{1}}^{t_{2}}\left|\left(T_{\gamma} u\right)^{\prime}(s)\right| d s \leq N^{\prime}\left(t_{2}-t_{1}\right)
$$

so $T_{\gamma}(\Omega)$ is an equicontinuous set in $X$.

Thus, by virtue of the Arzelà-Ascoli theorem, we deduce that the set $\overline{T_{\gamma}(\Omega)}$ is relatively compact, i.e., $T: K_{\gamma} \rightarrow K_{\gamma}$ is a compact operator.

Suppose that the first situation holds:

(i) (sublinear case) $f_{0}=\infty$ and $f^{\infty}=0$.

Since $f_{0}=\infty$, there exists a constant $\rho_{1}$ such that $f(t, u) \geq \delta_{1} u$, for all $t \in[1 / 2,1]$ and $0 \leq$ $u \leq \rho_{1}$, where $\delta_{1}$ is such that

$$
\frac{\delta_{1}}{C_{1}} \max _{t \in[0,1]}\left\{\int_{\frac{1}{2}}^{1} h_{1}(s) G_{m}(t, s) d s\right\} \geq 1
$$

Let $u \in K_{\gamma}$ with $\|u\|=\rho_{1}$, then from the previous expression we deduce

$$
\begin{aligned}
\left\|T_{\gamma} u\right\| & =\max _{t \in[0,1]}\left\{\int_{0}^{1} G_{m}(t, s) f(s, u(s)) d s\right\} \geq \max _{t \in[0,1]}\left\{\int_{\frac{1}{2}}^{1} G_{m}(t, s) f(s, u(s)) d s\right\} \\
& \geq \max _{t \in[0,1]}\left\{\int_{\frac{1}{2}}^{1} G_{m}(t, s) \delta_{1} u(s) d s\right\} \geq \delta_{1} \max _{t \in[0,1]}\left\{\int_{\frac{1}{2}}^{1} G_{m}(t, s) \frac{h_{1}(s)}{C_{1}}\|u\| d s\right\} \\
& =\|u\| \frac{\delta_{1}}{C_{1}} \max _{t \in[0,1]}\left\{\int_{\frac{1}{2}}^{1} h_{1}(s) G_{m}(t, s)\right\} \geq\|u\| .
\end{aligned}
$$

On the other hand, the continuity of $f$ in the second variable allows us to define the function

$$
\widetilde{f}(t, u)=\max _{z \in[0, u]}\{f(t, z)\}, \quad t \in[0,1], u \in \mathbb{R}
$$

which is monotone nondecreasing on $[0, \infty)$ for every $t \in[0,1]$. Now, since $f^{\infty}=0$, it follows (see [11]) that

$$
\lim _{u \rightarrow \infty}\left\{\max _{t \in[0,1]} \frac{\tilde{f}(t, u)}{u}\right\}=0
$$


Let us now take $\delta_{2}>0$ such that

$$
\delta_{2} \max _{t \in[0,1]}\left\{\int_{0}^{1} G_{m}(t, s) d s\right\} \leq 1 .
$$

From (23), we know that there exists $\rho_{2} \in \mathbb{R}, \rho_{2}>\rho_{1}>0$ such that $\tilde{f}(t, u) \leq \delta_{2} u$, for all $t \in[0,1]$ and $u \geq \rho_{2}$.

Let $u \in K_{\gamma}$ be such that $\|u\|=\rho_{2}$, then using the definition of $\tilde{f}$ and the above inequality we have

$$
\begin{aligned}
\left\|T_{\gamma} u\right\| & =\max _{t \in[0,1]}\left\{\int_{0}^{1} G_{m}(t, s) f(s, u(s))\right\} \leq \max _{t \in[0,1]}\left\{\int_{0}^{1} G_{m}(t, s) \tilde{f}(s, u(s))\right\} \\
& \leq \max _{t \in[0,1]}\left\{\int_{0}^{1} G_{m}(t, s) \tilde{f}(s,\|u\|)\right\} \leq \max _{t \in[0,1]}\left\{\int_{0}^{1} G_{m}(t, s) \delta_{2}\|u\|\right\} \\
& \leq\|u\| \delta_{2} \max _{t \in[0,1]}\left\{\int_{0}^{1} G_{m}(t, s)\right\} \leq\|u\| .
\end{aligned}
$$

Finally, the first part of Theorem 2 implies that there is at least one positive solution of problem (1)-(2), with $\gamma=m^{2}>0, u \in K_{\gamma}$, such that $\rho_{1} \leq\|u\| \leq \rho_{2}$.

Let us now consider the second case:

(ii) (superlinear case) $f^{0}=0$ and $f_{\infty}=\infty$.

Let $\delta_{2} \in(0,1]$, chosen in the same way as in the previous case. Since $f^{0}=0$, there exists a constant $r_{1}>0$ such that $f(t, u) \leq \delta_{2} u$ for all $t \in[0,1]$ and $0 \leq u \leq \delta_{2}$.

Let us choose $u \in K_{\gamma}$ be such that $\|u\|=r_{1}$, then

$$
\begin{aligned}
\left\|T_{\gamma} u\right\| & =\max _{t \in[0,1]}\left\{\int_{0}^{1} G_{m}(t, s) f(s, u(s))\right\} \leq \max _{t \in[0,1]}\left\{\int_{0}^{1} G_{m}(t, s) \delta_{2} u(s)\right\} \leq \\
& \leq\|u\| \delta_{2} \max _{t \in[0,1]}\left\{\int_{0}^{1} G_{m}(t, s)\right\} \leq\|u\| .
\end{aligned}
$$

Let us take $\delta_{3}>0$ so that

$$
\frac{\delta_{3}}{C_{1}} \max _{t \in[0,1]}\left\{\int_{\frac{1}{2}}^{1} h_{1}(s) G_{m}(t, s) d s\right\} \geq 1
$$

Since $f_{\infty}=\infty$, there exists $r_{2}>r_{1}>0$ with $C_{1} r_{2}>\underline{M} r_{1}$ (where $\underline{M}=\min _{t \in\left[\frac{1}{2}, 1\right]} h_{1}(s)$ ), so that $f(t, u) \geq \delta_{3} u$ for all $t \in[1 / 2,1]$ and $u \geq r_{2}$. Let $u \in K_{\gamma}$ be such that $\|u\|=r_{2} \frac{C_{1}}{\underline{M}}$, then, by the definition of $K_{\gamma}, u(t) \geq r_{2}$ for all $t \in[1 / 2,1]$ is satisfied.

From this, we deduce the following inequalities:

$$
\begin{aligned}
\left\|T_{\gamma} u\right\| & =\max _{t \in[0,1]}\left\{\int_{0}^{1} G_{m}(t, s) f(s, u(s)) d s\right\} \geq \max _{t \in[0,1]}\left\{\int_{\frac{1}{2}}^{1} G_{m}(t, s) f(s, u(s)) d s\right\} \\
& \geq \max _{t \in[0,1]}\left\{\int_{\frac{1}{2}}^{1} G_{m}(t, s) \delta_{3} u(s) d s\right\} \geq \delta_{3} \max _{t \in[0,1]}\left\{\int_{\frac{1}{2}}^{1} G_{m}(t, s) \frac{h_{1}(s)}{C_{1}}\|u\| d s\right\} \\
& =\|u\| \frac{\delta_{3}}{C_{1}} \max _{t \in[0,1]}\left\{\int_{\frac{1}{2}}^{1} h_{1}(s) G_{m}(t, s) d s\right\} \geq\|u\| .
\end{aligned}
$$


Finally, applying part 2 of Theorem 2, we conclude that problem (1)-(2), with $\gamma=m^{2}>0$, has at least one positive solution $u \in K_{\gamma}$ such that

$$
r_{1} \leq\|u\| \leq \frac{C_{1}}{\underline{M}} r_{2}
$$

The cases $\gamma=0$ and $\gamma=-m^{2}<0$ can be proved in a similar way.

Remark 15 It should be noted that, since $G(0, s)=0$, in order to ensure the existence of $r_{2}$ in the case (ii) of Theorem 14, we have reduced the interval considered in the definition of the limits $f_{0}$ and $f_{\infty}$ from $[0,1]$ to $[1 / 2,1]$.

In fact, it is enough to take $a, b \in \mathbb{R}$ such that $0<a<b \leq 1$ and redefine the limits as

$$
f_{0}=\lim _{u \rightarrow 0^{+}}\left\{\min _{t \in[a, b]} \frac{f(t, u)}{u}\right\} \text { and } f_{\infty}=\lim _{u \rightarrow+\infty}\left\{\min _{t \in[a, b]} \frac{f(t, u)}{u}\right\} .
$$

In this case, it is easy to check that Theorem 14 remains true for solutions defined in cone $K_{\gamma}$, given by expressions (19), (20) and (21), by replacing on their definitions $[1 / 2,1]$ by $[a, b]$.

Remark 16 Let us now consider problem (1) coupled to the boundary conditions

$$
u(0)=\lambda \int_{0}^{1} u(s) d s, \quad u(1)=0 .
$$

It is easy to check that $v(t):=u(1-t), t \in[0,1]$, also satisfies (1) together with

$$
v(0)=u(1)=\lambda \int_{0}^{1} u(s) d s=\lambda \int_{0}^{1} v(s) d s, \quad v(1)=u(0)=0 .
$$

So, if we denote

$$
f_{0}^{\prime}=\lim _{u \rightarrow 0^{+}}\left\{\min _{t \in\left[0, \frac{1}{2}\right]} \frac{f(t, u)}{u}\right\} \text { and } f_{\infty}^{\prime}=\lim _{u \rightarrow+\infty}\left\{\min _{t \in\left[0, \frac{1}{2}\right]} \frac{f(t, u)}{u}\right\}
$$

and, if $\gamma=0$ :

$$
K_{\gamma}^{\prime}=\left\{u \in X ; u(t) \geq 0, t \in[0,1], u(t) \geq(1-t) \frac{\lambda}{2}\|u\|, t \in[0,1]\right\} .
$$

If $\gamma>0$ :

$$
K_{\gamma}^{\prime}=\left\{u \in X ; u(t) \geq 0, u(t) \geq \frac{h_{1}(1-t)}{C_{1}}\|u\|, t \in[0,1]\right\}
$$

If $\gamma<0$ :

$$
K_{\gamma}^{\prime}=\left\{u \in X ; u(t) \geq 0, u(t) \geq \frac{h_{2}(1-t)}{C_{2}}\|u\|, t \in[0,1]\right\}
$$

Thus, the following result is then obtained. 
Corollary 17 Consider problem (1), (24) and let $\Delta:\left(-\infty, \pi^{2}\right) \rightarrow \mathbb{R}$ be the function defined in (22). Suppose further that $(f)$ holds and one of the following conditions is satisfied:

(i) (sublinear case) $f_{0}^{\prime}=\infty$ and $f^{\infty}=0$.

(ii) (superlinear case) $f^{0}=0$ and $f_{\infty}^{\prime}=\infty$.

So, for all $\gamma<\pi^{2}$ and $0<\lambda<\Delta(\gamma)$ there is a positive solution of problem (1), (24), $u \in K_{\gamma}^{\prime}$.

Moreover, analogous considerations to Remark 15 remain valid for problem (1), (24).

\subsection{Examples}

Example 18 Consider problem (1)-(2) with

$$
f(t, x)=\sqrt[5]{x^{3}+x}+\log (3 t+x) .
$$

It is easy to verify that, for $u>0$,

$$
\min _{t \in\left[\frac{1}{2}, 1\right]} \frac{f(t, u)}{u}=\frac{\sqrt[5]{u^{3}+u}+\log \left(\frac{3}{2}+u\right)}{u} \text { and } \max _{t \in[0,1]} \frac{f(t, u)}{u}=\frac{\sqrt[5]{u^{3}+u}+\log (3+u)}{u} .
$$

Taking limits, it is easy to check that $f_{0}=\infty$ and $f^{\infty}=0$, i.e., we are in the sublinear case. Since $f$ fulfills $(f)$, we have the hypotheses of the case (i) Theorem 14, so we can ensure the existence of a positive solution for problem (1)-(2).

Example 19 Consider again the problem (1)-(2) with

$$
f(t, x)=t x^{3}+e^{t x}-1
$$

In this case, we have for all $u>0$

$$
\min _{t \in\left[\frac{1}{2}, 1\right]} \frac{f(t, u)}{u}=\frac{u^{2}}{2}+\frac{e^{\frac{u^{2}}{2}}-1}{u} \text { and } \max _{t \in[0,1]} \frac{f(t, u)}{u}=u^{2}+\frac{e^{u^{2}}-1}{u} .
$$

Again, taking limits we see that $f^{0}=0$ and $f_{\infty}=\infty$, that is, we have the superlinear case. As $f$ verifies $(f)$, the second part of Theorem 14 guarantees the existence of a positive solution of problem (1)-(2).

\section{Acknowledgements}

The authors thank the reviewers of the manuscript for their comments and suggestions, which have contributed to improving the work.

Funding

Alberto Cabada was partially supported by Xunta de Galicia (Spain), project EM2014/032 and AlE, Spain and FEDER, grant MTM2016-75140-P.

Availability of data and materials

Not Applicable.

Competing interests

The authors declare that they have no competing interests.

Authors' contributions

The two authors have contributed equally and significantly in writing this article. All the authors read and approved the final manuscript. 


\section{Publisher's Note}

Springer Nature remains neutral with regard to jurisdictional claims in published maps and institutional affiliations.

Received: 16 April 2021 Accepted: 19 June 2021 Published online: 29 July 2021

\section{References}

1. Ahmad, B., Hamdan, S., Alsaedi, A., Ntouyas, S.K.: On a nonlinear mixed-order coupled fractional differential system with new integral boundary conditions. AIMS Math. 6(6), 5801-5816 (2021)

2. Cabada, A.: Green's Functions in the Theory of Ordinary Differential Equations. Springer Briefs Math. Springer, New York (2014)

3. Cabada, A., Cid, J.Á., Máquez-Villamarín, B.: Computation of Green's functions for boundary value problems with mathematica. Appl. Math. Comput. 219(4), 1919-1936 (2012)

4. Cabada, A., Cid, J.Á., Máquez-Villamarín, B.: Green's Function Computation. (Mathematica Package), 2014. https://library.wolfram.com/infocenter/MathSource/8825/

5. Cabada, A., Hamdi, Z:: Nonlinear fractional differential equations with integral boundary value conditions. Appl. Math. Comput. 228, 251-257 (2014)

6. Cabada, A., Jebari, R.: Existence results for a clamped beam equation with integral boundary conditions. Electron. J. Qual. Theory Differ. Equ. 2020, Article ID 70 (2020)

7. Chandran, K., Gopalan, K., Tasneem, Z.S., Abdeljawad, T:: A fixed point approach to the solution of singular fractional differential equations with integral boundary conditions. Adv. Differ. Equ. 2021, Article ID 56 (2021)

8. Duraisamy, P., Nandha, G.T., Subramanian, M.: Analysis of fractional integro-differential equations with nonlocal Erdélyi-Kober type integral boundary conditions. Fract. Calc. Appl. Anal. 23(5), 1401-1415 (2020)

9. Hu, Q.-Q., Yan, B.: Existence of multiple solutions for second-order problem with Stieltjes integral boundary condition. J. Funct. Spaces 2021, Article ID 6632236 (2021)

10. Krasnosel'skiĭ, M.A.: Positive Solutions of Operator Equations. Noordhoff, Groningen (1964)

11. Wang, H.: On the number of positive solutions of nonlinear systems. J. Math. Anal. Appl. 281(1), 287-306 (2003)

12. Zhang, Y., Abdella, K., Feng, W.: Positive solutions for second-order differential equations with singularities and separated integral boundary conditions. Electron. J. Qual. Theory Differ. Equ. 2020, Article ID 75 (2020)

\section{Submit your manuscript to a SpringerOpen ${ }^{\circ}$ journal and benefit from:}

- Convenient online submission

- Rigorous peer review

- Open access: articles freely available online

- High visibility within the field

- Retaining the copyright to your article 